\title{
Los desahucios en la prensa española: distintos relatos sobre los asuntos públicos
}

\author{
Palmira CHAVERO* \\ pchavero@gmail.com
}

(Abstracts y palabras clave al final del artículo)

Enviado: 20 de abril de 2013

Evaluado: 26 de junio de 2013

Aceptado: 21 de julio de 2013

\section{LA ESFERA PÚBLICA EN ESPAÑA: LOS MEDIOS DE COMUNICACIÓN}

Siguiendo la propuesta de Hallin y Mancini (2004), España formaría parte del grupo de países pertenecientes al sistema mediático de Pluralismo Polarizado o Mediterráneo, caracterizado por una serie de rasgos propios. Resumiendo mucho la propuesta de estos autores, podemos identificar las siguientes características del caso español. En primer lugar, fruto del tardío nacimiento de esta industria, la prensa española cuenta con tiradas pequeñas y enfocadas sobre todo a las elites, principales consumidoras mediáticas. En segundo lugar, existe una estrecha relación entre los partidos políticos y los medios de comunicación; es lo que los autores denominan "paralelismo político", una relación según la cual no podemos hablar de prensa de partidos, pero sí de un alineamiento ideológico de los medios con las distintas opciones políticas. En tercer lugar, los profesionales del periodismo en España disponen de un bajo nivel de autonomía, a diferencia de lo que sucede con otros agentes, como los lobbies y asociaciones sectoriales, en otros países; aunque proliferan las asociaciones profesionales periodísticas, su capacidad y herramientas de actuación en la práctica son muy limitadas. Por último, el Estado interviene en el sistema mediático por una doble vía: directa - a través de la propiedad de la radio y televisión pública- e indirecta -mediante la concesión de licencias audiovisuales de funcionamiento-. Esta caracterización del sistema mediático español acentúa la importancia de los medios de comunicación a la hora de informar sobre los asuntos públicos $\mathrm{y}$, en última instancia, conformar un modelo u otro de opinión pública.

En lo que en este trabajo nos interesa, esta participación de los medios en la esfera pública tiene su interés en la manera en que los medios de comunicación cons-

\footnotetext{
* Colaboradora honorífica del departamento de Sociología VI (Opinión Pública y Cultura de Masas), en la Facultad de Ciencias de la Información de la UCM
} 
truyen el relato de determinados asuntos públicos y, con ello, influyen en un proceso que puede llegar a culminar en la toma de decisiones políticas con respecto al tema tratado. En todo caso, hay que tener en cuenta que la construcción del relato ciudadano sobre un asunto determinado es producto de la interacción de diversas agendas: política, mediática, pública y personal. En este sentido, cabe esperar que en aquellos temas con los que el ciudadano tenga experiencia propia (tema obtrusive), esta tendrá un peso mayor en la opinión que el individuo se forme con respecto al tema y, en consecuencia, utilizará la propuesta mediática para completar (o rechazar) su opinión sobre el asunto.

La característica principal a lo largo de los últimos años ha sido que todas las agendas mencionadas han estado hegemonizadas por la crisis económica y sus consecuencias. En el caso que nos ocupa, nos centramos en el análisis de una de las últimas consecuencias de la crisis económica: los desahucios. En concreto, realizamos un estudio sobre la manera en que los medios de comunicación han tratado este tema desde su entrada en la agenda mediática hasta su desactivación.

\section{OBJETO DE ESTUDIO Y METODOLOGÍA}

Para abordar este trabajo intentamos dar respuesta a una serie de preguntas de investigación: ¿Cómo se convierten los desahucios en issue? ¿Construye la prensa de referencia relatos distintos del mismo tema? ¿Cómo los construye, en tal caso? ¿Responden los relatos mediáticos a posturas ideológicas polarizadas? ¿Existe algún tipo de interacción entre los medios de comunicación y el sistema político? ¿Cómo se desactiva el issue?

A fin de dar respuesta a estas y otras preguntas de investigación, realizamos un estudio del framing y un análisis crítico de discurso de los dos diarios españoles de referencia (El País y El Mundo) a lo largo del último trimestre de 2012. En concreto, comenzamos el período de estudio el 1 de octubre de 2012, lo que nos permite analizar el momento en el que los desahucios aparecen en la agenda mediática y la manera en que se produce tal aparición. El período de estudio finaliza el 31 de diciembre de 2012, una vez aprobado (en noviembre) el decreto ley que pretende regular esta materia. Si bien la mayor parte de la actividad mediática, social y política de nuestro objeto de estudio se produce a lo largo del mes de noviembre, extender el análisis durante los meses previo y posterior nos permite vislumbrar las estrategias mediáticas y analizar los precedentes y consecuencias de las primeras medidas políticas tomadas en materia de desahucios, todo lo cual es fundamental para analizar las posibles influencias entre los distintos actores.

Para abordar nuestro objeto de estudio, analizamos las portadas y editoriales de El País y El Mundo, tomando aquellas unidades informativas referidas a los desahucios en el período citado. La selección de estos dos diarios responde, por un lado, a su consideración como prensa de referencia (Reig, 1998) y, por otro, al diferente posicionamiento ideológico en el que se ubican, de tal manera que el estudio de ambos arroja una visión complementaria del escenario mediático; son, asimismo, los diarios de mayor tirada. Una vez obtenido el corpus informativo, estudiamos los 
encuadres (frames) de cada uno de los diarios, que completamos con un análisis de discurso. Para realizar el estudio del framing, partimos de la propuesta de Entman, según la cual enmarcar supone "seleccionar y destacar algunos aspectos de una realidad percibida y realzar la relevancia de una interpretación y evaluación de esa realidad" (Entman, 2004: 26). Un frame o encuadre, conlleva, por tanto, la propuesta de tres elementos principales: diagnóstico de la situación, atribución de responsabilidad y propuesta de solución. El análisis de todas las portadas y editoriales del período analizado nos permitirá identificar todos estos elementos, que presentaremos con una formulación narrativa que concentra el encuadre con el que cada uno de los diarios presenta el issue "desahucios". A la hora de realizar la citada formulación del encuadre, nos ajustaremos al lenguaje del propio medio, tomando en la medida de lo posible construcciones publicadas por tal medio.

En segundo lugar, sobre el mismo corpus informativo realizamos un análisis crítico del discurso, para lo que partimos de los parámetros propuestos por Van Dijk (1996) y, a partir de las estructuras discursivas, identificaremos la ideología subyacente de cada uno de los diarios. En concreto, realizamos un análisis exhaustivo de las informaciones recopiladas prestando especial atención a determinados aspectos que propone este autor: elementos léxicos, descripciones y proposiciones, entre otros. Dentro de estas últimas, prestaremos especial atención al "cuadrado ideológico", estructura según la cual los medios tienden a destacar las buenas acciones propias y las acciones/atributos negativos del adversario, al tiempo que se reduce la visibilidad de las acciones propias cuando son negativas y las del adversario cuando se trata de acciones/propiedades positivas. Hay que tener en cuenta, sin embargo, la predisposición de la prensa española al negativismo (Castromil, 2012; González y Chavero, 2013). A partir de aquí, estableceremos categorías de análisis propias aplicables a nuestro objeto de estudio que nos permitan identificar el posicionamiento de cada uno de los medios.

\section{LA CRISIS ECONÓMICA COMO CONTEXTO}

Antes de analizar el issue "Desahucios", conviene repasar brevemente el recorrido de la crisis económica en los últimos años tal y como la han mostrado los medios de comunicación en España. Aunque algunos indicadores económicos alertaban ya en 2007 de una recesión económica, fue la caída de Lehman Brothers (septiembre 2008) el momento en el que la crisis económico-financiera explotaba a nivel internacional; en el caso español, la situación se vio agravada por la explosión de una crisis económica de carácter interno provocada por la burbuja inmobiliaria de los años anteriores. Tras un primer intento de lograr una salida social a la crisis, el gobierno socialista de Rodríguez Zapatero acata en mayo de 2010 las exigencias internacionales de austeridad y aprueba los primeros recortes sociales, con lo que el coste de la crisis comienza a recaer sobre la clase media. Ante el deterioro progresivo del contexto económico y político (el gobierno socialista pierde las elecciones municipales y autonómicas de mayo de 2011 por una diferencia de casi 10 puntos), Zapatero ade- 
lanta las elecciones generales, que dan el poder al PP el 20 de noviembre de 2011 con una holgada mayoría absoluta en el Parlamento.

Para entonces, la crisis económica había tenido un segundo repunte (verano 2011), que llevó al PSOE y PP al único acuerdo alcanzado en la pasada legislatura: la reforma de la Constitución, que daba prioridad al pago de la deuda a los inversores y limitaba el déficit público. Entretanto, el empeoramiento de los datos económicos incrementaba la percepción negativa del clima económico por parte de la ciudadanía. Con la llegada del Partido Popular al gobierno, las medidas de austeridad (y con ellas, los recortes a las clases medias) se incrementan significativamente; en términos económicos, las consecuencias de las medidas tienen su reflejo en un crecimiento progresivo del desempleo y la disminución del poder adquisitivo (y, por tanto, de consumo y de ahorro) de las familias. En el plano social, se produce un aumento de las protestas sociales, una mayor desafección de los ciudadanos hacia la clase política, una nueva huelga general y el empeoramiento generalizado del clima social y político.

Además de este somero recorrido por la evolución de la crisis, conviene atender a la manera en que el tema ha sido presentado por los medios a la audiencia, para lo cual recurrimos al análisis del framing. En este sentido, la evolución de la crisis ha sido como sigue en un primer momento, predomina el encuadre de la "salida social a la crisis", según la cual el coste de la salida a la crisis no recaería sobre los ciudadanos. La discusión en esta fase está presidida por sucesivos intentos de alcanzar un gran pacto político y socioeconómico que, sin embargo, no llega a fructificar. En un segundo momento, los ajustes acometidos por el gobierno socialista en mayo de 2010 hacen saltar por los aires el encuadre de la salida social, por cuanto la clase media comienza a sufrir los primeros recortes. El problema para el gobierno socialista es que no cuenta con un encuadre alternativo, de manera que se debate entre continuar con una agenda reformista o recuperar temas de la agenda social que había caracterizado su etapa anterior. La convocatoria de la huelga general rompe definitivamente el encuadre de la salida social y, tras el relativo fracaso del paro, el Gobierno continúa las reformas iniciadas hasta que, en enero de 2011, logra el Acuerdo Social y Económico (González y Chavero, 2012: 302 ss).

Sin embargo, el declive electoral del gobierno de Zapatero en mayo de 2011 y el repunte de la crisis económica ese mismo verano agitan de nuevo el clima social y político, que se mantendrá en la medida en que el nuevo gobierno "popular" de Rajoy profundiza en los recortes sociales sin que ello se refleje en mejoras económicas.

\section{LOS DESAHUCIOS EN LOS MEDIOS}

La cara más visible de la crisis económica la conforma el progresivo crecimiento del desempleo, que alcanza el $26,02 \%$ en el cuarto trimestre de 2012 y que, en conjunción con otros factores económicos, origina en último término la disminución del poder adquisitivo de las familias. Esta pérdida de capacidad económica, combinada con un precio de la vivienda fruto de la burbuja inmobiliaria, acaba por generar una de las últimas consecuencias sociales en la cadena de la crisis: los desahu- 
cios, esto es, el desalojo de la vivienda por parte de los bancos ante el impago de las cuotas hipotecarias.

En términos legislativos, la Ley Hipotecaria ${ }^{1}$ (de 8 de febrero de 1946, vigente desde 19 de marzo de 1946) es muy clara en su amparo al acreedor:

Art.127: [...] Al vencimiento del plazo para el pago de la deuda, el acreedor podrá pedir que se despache mandamiento de ejecución contra todos los bienes hipotecados, estén o no en poder de uno o varios terceros poseedores; pero estos no podrán ser requeridos al pago, sino después de haberlo sido el deudor y no haberlo realizado.

Será juez o tribunal competente para conocer del procedimiento el que lo fuera respecto del deudor. No se suspenderá en ningún caso el procedimiento ejecutivo por las reclamaciones de un tercero, si no estuvieren fundadas en un título anteriormente inscrito, ni por la muerte del deudor o del tercer poseedor.

Estas exigencias legales se han venido cumpliendo desde los inicios de la crisis económico-financiera. No en vano, en el período comprendido entre 2007 y el primer trimestre de 2011 se iniciaron 300.000 ejecuciones hipotecarias ${ }^{2}$, cifra que continuó aumentando durante 2011 y 2012. Hasta septiembre de 2012 se produjeron 49.702 lanzamientos notificados y 76.724 lanzamientos acordados por juzgados de primera instancia.

Lejos todavía del foco de los medios de comunicación, la preocupación ciudadana por los desahucios ha tenido un desarrollo paralelo a la crisis. Las primeras movilizaciones sociales por este motivo comienzan en 2007 y una de las reivindicaciones iniciales del movimiento social 15M era la dación en pago; la principal asociación en la lucha contra los desahucios, la Plataforma de Afectados por las Hipotecas $\left(\mathrm{PAH}^{3}\right)$, tiene un papel muy destacado tanto dentro como fuera del $15 \mathrm{M}$. Hasta principios de 2013, las campañas y actuaciones de esta plataforma ciudadana consiguieron detener 523 desalojos. En un intento por recoger esta preocupación ciudadana, el grupo mixto ICV-IU-ERC había llevado a debate al Congreso de los Diputados este tema el 16 de junio de 2010, si bien la iniciativa quedó bloqueada.

A pesar de la preocupación social y -en menor medida- política por este tema, los desahucios no se transforman en issue hasta el mes de noviembre de 2012. ¿Qué

\footnotetext{
1 http://noticias.juridicas.com/base_datos/Privado/lh.t5.html.

2 Datos del Consejo General del Poder Judicial, disponibles en http://afectadosporlahipoteca.com/ nuevo-record-de-desahucios-en-espana-datos-cgpj/

3 "La PAH es un movimiento apartidista, de auto organización ciudadana y plural, y cuya única relación con los partidos políticos se ha limitado a interpelarles, a todos por igual, para pedirles que escuchen y apoyen las propuestas de la ciudadanía para cambiar una situación de flagrante injusticia social. Si bien es cierto que algunos partidos (como ICV, IU o ERC) han respondido mucho más a las interpelaciones que otros (básicamente PP y PSOE), la PAH ha insistido desde sus comienzos en su carácter apartidista, el cual ha permitido que miles de personas se sumen a un movimiento, cuyas demandas cuentan, a día de hoy, con un amplio consenso social. La PAH, avalada por su trayectoria, considera imprescindible mantener este carácter apartidista para continuar sumando esfuerzos frente a la vulneración sistemática del derecho a la vivienda y el drama social al que se ven abocadas miles de familias en el Estado Español. " (http://afectadosporlahipoteca.com/la-pah-desmiente-acuerdo-en-X-asamblea-de-iu-la-pah-es-apartidista/ a raíz de los vínculos que los medios le atribuyen con IU tras la asamblea federal celebrada en Madrid entre el 14 y el 16 de diciembre de 2012).
} 
lo convierte, entonces, en un tema mediático? Una de las justificaciones que se argumentan para este salto a la esfera mediática suele ser la cercanía temporal de dos suicidios (uno en Granada y otro en Barakaldo). Sin embargo, según los datos del Instituto Nacional de Estadística, desde 2011 se producen una media de tres suicidios diarios por motivos económicos, habiéndose convertido esta en la primera causa de muerte no natural del país. ¿Qué factores, por tanto, estarían contribuyendo a convertir a los desahucios en un tema mediático?

En primer lugar, cabe tener en cuenta la constante presión social de los últimos años a través de manifestaciones y diferentes formas de protesta (flashmobs en bancos y establecimientos, apoyo y protección de familias que van a ser desahuciadas, etc.). En segundo lugar, en la primera quincena de noviembre (2012) se dan a conocer dos informes judiciales instando a la modificación de la Ley Hipotecaria por el trato injusto que supone para los ciudadanos españoles; el primero de ellos procede del Consejo General del Poder Judicial y el segundo del Tribunal de Justicia de la Unión Europea. La aparición de los actores judiciales genera la reacción de la prensa española, que demanda una reforma legislativa con diferente grado de exigencia ("No vale abstenerse", editorial El País 26/X/2012; "La UE cuestiona los desahucios", editorial El Mundo 09/XI/2012). En tercer lugar, la confluencia de los dos suicidios anteriormente citados, que saltan a la esfera mediática motivados por tres factores: a) la cercanía temporal de los dos casos, que activa la alarma social, b) el momento en el que se producen los suicidios (justo antes del desahucio, con la llegada de la Policía y los jueces) y c) la condición política de una de las víctimas, exconcejala del Partido Socialista de Euskadi.

En ese momento, el suicidio, hasta entonces tema tabú en los medios de comunicación, entra en la agenda mediática de la mano de los desahucios. De esta manera, los desahucios se convierten en issue, cumpliendo con algunos de los factores de noticiabilidad: a) personalización (se le pone rostro a la crisis en sus consecuencias más graves), b) negatividad (la muerte, probablemente la máxima expresión de negatividad para la cultura occidental), c) sorpresa (dos casos muy cercanos en el tiempo) y d) espectacularización (suicidio en el momento del desalojo).

Los citados están entre los cinco factores principales de noticiabilidad de los que nos habla la literatura académica (Schulz, citado por Donsbach en Muñoz Alonso y Rospir, 1995: 45):

a) Temática. La relevancia de los acontecimientos y, por tanto, su probabilidad de convertirse en noticia y su rango en la agenda, está influida por el tema tratado, prioridad esta que responde a la agenda temática que plantean los medios de comunicación. Los asuntos económicos han sido en los últimos años los principales en la agenda mediática española.

b) Negatividad. La prensa española tiene una comprobada proclividad hacia un tono negativo, aumentando por tanto la probabilidad de que un acontecimiento se convierta en issue si tiene un cariz negativo o refleja conflicto entre los actores implicados.

c) Personalización. Una noticia tendrá más posibilidades de aparecer en los medios cuando tenga un protagonista reconocible, que guíe la acción y con el 
que se pueda llegar a identificar la responsabilidad de su éxito/fracaso si se trata de un actor político.

d) Proximidad geográfica. La zona de difusión del medio de comunicación influye en la selección de los acontecimientos que se convierten en noticia, debido a la vinculación de la audiencia.

e) Sorpresa. Utilizada como estrategia para captar y mantener la atención de la audiencia, la sorpresa cobra importancia a la hora de primar un acontecimiento sobre otro y de darle una determinada cobertura.

f) Espectacularización/ dramatización. En la medida en que la prensa busca hechos que se salgan de la norma ("La noticia es que un hombre muerda a un perro, no a la inversa", reza una máxima periodística), los hechos con rasgos de dramatización y espectacularización estarían más cerca de convertirse en noticia que aquellos anodinos.

\section{EL ESTUDIO DEL FRAMING EN LA PRENSA ESPAÑOLA}

Una vez que los desahucios se convierten en issue, regresamos a otra de nuestras preguntas de investigación iniciales: ¿Cómo han tratado los medios de comunicación este tema? Para responder a esta pregunta, realizamos un análisis de los dos diarios españoles principales: El País (progresista) y El Mundo (conservador), con los que cubrimos los dos polos ideológicos de la prensa de referencia.

A partir de los datos analizados, podemos formular el frame con el que El País aborda los desahucios como sigue: "Los desahucios son una de las consecuencias más graves de la crisis económica y suponen un drama social que requiere de una reforma estructural urgente. El decreto aprobado por el Gobierno es un primer paso ante la demanda social y judicial que debe tener una continuación urgente y profunda".

Por su parte, el encuadre de El Mundo puede quedar establecido en los siguientes términos: "Los desahucios constituyen un grave problema social que requiere soluciones. El PSOE, que no actuó cuando estaba gobernando, dificulta ahora el pacto con el PP para aliviar este drama. La lucha más eficaz contra los desahucios es colocar al país en condiciones de crecer".

Si bien ambos marcos comparten elementos (gravedad de la situación en el diagnóstico), la principal diferencia entre ellos la encontramos en otro de los elementos que componen el encuadre: la atribución de responsabilidad, que recae sobre el partido de la oposición (PSOE) para el diario conservador, al que responsabiliza de no haber actuado a tiempo y, después, dificultar el consenso. El otro elemento diferenciador es la solución propuesta por cada uno de ellos: el diario progresista insta a una profunda reforma legislativa, que habría de nacer de un gran pacto entre los principales partidos. El Mundo, sin embargo, confía en la recuperación económica para sanear la situación, recuperación que vendría en todo caso de la actuación del gobierno "popular".

En un análisis retrospectivo, podemos recordar los encuadres con los que cada uno de estos medios ha abordado los problemas económicos en los últimos años (Chavero, 2012: 319), lo que nos ayuda a entender los actuales encuadres sobre los desahucios. Durante 2009 y parte de 2010, El País exoneró de su responsabilidad en 
la crisis al gobierno de Zapatero, presentándola como una crisis internacional. Eso no era óbice, sin embargo, para reconocer la lentitud y la improvisación socialista a la hora de actuar. La salida a la crisis se complicaba en la medida en que la oposición "popular" se limitaba a un comportamiento electoralista, según el encuadre del grupo PRISA. Frente a este marco, El Mundo defendía que se trataba de una crisis internacional agravada por un gobierno socialista incompetente, que además se estaría empeñando en marginar a la oposición.

El Acuerdo Social y Económico firmado a principios de 2011 dio argumentos a El País para reforzar su apoyo al gobierno socialista, en un encuadre según el cual el Gobierno, que siempre habría llevado la iniciativa, ha de mantenerse firme para garantizar la estabilidad. Para El Mundo, sin embargo, el proyecto político de Zapatero en 2011 ya está agotado y estaríamos ante un gobierno incapaz de ofrecer soluciones a la crisis, para la cual la única solución pasaría por un adelanto electoral que dejase paso a un gobierno del PP.

Este análisis nos permite comprobar cómo ambos diarios, al abordar los desahucios, dan continuidad a los encuadres mantenidos durante los años previos en materia económica, en los que cada una de las cabeceras realiza una atribución de responsabilidad distinta y, en consecuencia, también propone soluciones dispares.

\section{EL DISCURSO CONSTRUIDO POR LOS MEDIOS}

Además de las diferencias de encuadre observadas, cada uno de los medios analizados ha construido un relato propio sobre los desahucios, lo que genera distintas maneras de proponer a la audiencia la manera de afrontar el debate sobre los asuntos de relevancia. Conviene recordar que en un sistema mediático de pluralismo polarizado como el español, el relato mediático no es casual, sino que responde al posicionamiento ideológico de los medios; para comprobar si esto es así, realizamos un análisis de discurso al corpus informativo que sometemos a estudio.

En primer lugar, El País ofrece un discurso que gira en torno a dos ejes temáticos: el drama social que suponen los desahucios y la reivindicación de la política como herramienta para solucionar los problemas ciudadanos, que tendría su traducción última en un acuerdo entre los grandes partidos. El Mundo, sin embargo, trata el tema en torno a ejes temáticos distintos: la irresponsabilidad de los ciudadanos y los bancos como origen del problema y el crecimiento económico del país como vía de solución, que confiaría en última instancia al gobierno. La particularidad con la que se encuentra El Mundo a la hora de construir su relato sobre los desahucios es que el diario sugiere la responsabilidad de los propios ciudadanos, pero lo hace en una situación en la que existe un amplio consenso en la opinión pública sobre la responsabilidad de los bancos y en la que, muy al contrario, los ciudadanos son las víctimas $^{4}$. Esto complica la construcción del relato para el diario conservador, que elude el enfrentamiento directo con una opinión pública homogénea.

\footnotetext{
4 "Vuestra crisis no la pagamos" es uno de los gritos más habituales en las movilizaciones sociales e ilustra claramente la situación expuesta.
} 
Además de los ejes temáticos sobre los que giran sendas construcciones mediáticas, conviene atender a otra categoría de análisis: la iniciativa que cada uno de los medios atribuye a los distintos actores. Por parte del diario progresista, el gobierno del PP se limita a reaccionar a una tragedia social y una presión procedente tanto del poder judicial como de la sociedad, que estarían llevando la iniciativa. Por el contrario, para El Mundo estamos ante una estrategia puramente demagógica del PSOE como respuesta al éxito del PP, que vendría a ofrecer soluciones reales a pesar de las dificultades políticas que la oposición le plantea.

La principal y más directa consecuencia de la presión social, judicial y mediática para la reforma de la Ley Hipotecaria es el breve proceso de negociación que abordan PP y PSOE, pero que finalmente no fructifica. Tras el fracaso del diálogo entre los dos principales partidos para aprobar medidas conjuntas sobre los desahucios, el PP aprueba un decreto ley con el que el Gobierno ofrece unas garantías mínimas a los colectivos más vulnerables de las que, en la práctica, los ciudadanos que se pueden beneficiar son una minoría. Los puntos principales del decreto ley 15 noviembre 2012 se resumen en:

- No se modifica la Ley Hipotecaria ni la de Enjuiciamiento Civil

- No se aprueba la dación en pago

- No se modifican intereses de demora

- Establece una moratoria de dos años para quien cumpla dos requisitos: 1. La renta anual de la unidad familiar sea inferior a $19.164 €$ y 2 . Sean familias numerosas o tengan dos hijos a cargo en familias monoparentales o un menor de tres años o un discapacitado o enfermo grave o parados que hayan agotado prestación.

Este proceso -y, en particular, unos resultados que en poco resuelven la demanda ciudadana- nos remite a lo que Meyer (2002) definió como "pseudopolítica", según la cual la política queda reducida a una actividad autorreferencial en la que los distintos actores debaten sobre la capacidad y la predisposición propias para solucionar los problemas pero que, en la práctica, no se traduce en ninguna acción concreta.

Una vez aprobada la solución gubernamental al problema de los desahucios, el papel de los medios de comunicación continúa (y finaliza) con la valoración de la medida. En este caso, si nos centramos en la información publicada por los citados medios el día posterior a la aprobación del decreto ley (16/XI/2012), encontramos de nuevo algunas diferencias entre ellos.

Por un lado, El País sitúa al decreto ley como noticia de apertura ("La moratoria en los desahucios se aplicará sólo en los casos extremos"// El Gobierno aprueba un decreto ley sin pactar con los socialistas/El umbral de renta para evitar el desalojo se fija en 19.164 euros), que acompaña con su respectivo editorial ("Un primer paso"//El decreto contra los desahucios debe continuarse con una revisión legal profunda) y su artículo de opinión más importante, "Cuarta página" ("Los desahucios: emergencia nacional"). Vemos, por tanto, cómo El País lo propone como tema más importante del día, destacando la ausencia de pacto a pesar de las negociaciones y los requisitos para beneficiarse de la reforma, sugiriendo la ineficacia del decreto ("El gobierno no aclara cuántas personas se beneficiarán") y advirtiendo de la necesidad de continuar el camino para solucionar el "grave problema social". 
Por otro lado, El Mundo, inmerso ya en su propia campaña electoral ante los comicios catalanes (que se celebrarían el 25 de noviembre) y cumpliendo con una de las pautas del cuadrado ideológico (destacar los atributos negativos del adversario), dedica su noticia de apertura del 16 de noviembre a la presunta corrupción de $\mathrm{CiU}$, relegando a los desahucios a un segundo plano, aunque también le dedica uno de sus editoriales ("Un paso adelante en la solución de los desahucios"). El Mundo presenta el decreto como un éxito del gobierno de Rajoy ("El Gobierno cumplió ayer con la promesa de su presidente..."), que estaría atendiendo a los intereses ciudadanos en detrimento de los de los bancos ("El Gobierno realojará a los desahuciados en pisos de los bancos", noticia secundaria de la portada), de esta manera destaca doblemente el éxito del PP: favorecer a los ciudadanos y no sucumbir a los bancos (señalados como los responsables de la crisis por los ciudadanos).

A partir de entonces $-\mathrm{y}$ a pesar de que el número de suicidios y de desalojos no se detiene- el tema desaparece del foco mediático, eliminando con ello la presión sobre los actores políticos en esta materia.

\section{CONCLUSIONES Y DISCUSIÓN}

Este trabajo nos ha permitido estudiar la aparición de un tema (los desahucios) en la agenda mediática para desactivar la alarma social, la manera de abordarlos por parte de los principales diarios y su desaparición de los medios. Además de la interacción que se produce entre los actores políticos y mediáticos, en este proceso participan otros agentes sociales, como las plataformas ciudadanas, el poder judicial y el policial (ante la negativa de una parte del cuerpo policial a participar de los desahucios, El Mundo responde con un editorial -"El SUP no debe amparar la desobediencia policial", $12 / \mathrm{XI} / 2012^{5}$ - en el que insta a la Policía a ejecutar las órdenes judiciales).

En primer lugar, comprobamos que la aparición del tema en la agenda mediática no responde a criterios de actualidad informativa, pero sí a otros factores de noticiabilidad a partir de los dos casos de suicidios: personalización (víctimas de la crisis), negatividad (muerte), sorpresa (dos casos muy cercanos en el tiempo que activan la alarma social) y espectacularización (momento en el que se producen los suicidios). A estos factores hay que añadir la presión judicial del CGPJ y del Tribunal Europeo. Cuando esta presión judicial se une a la presión social y, finalmente, la mediática, el gobierno del Partido Popular reacciona con una promesa de reforma de la ley hipotecaria y un (breve) proceso de negociación con el principal partido de la oposición,

5 [...] "La labor de la Policía es cumplir las órdenes de sus superiores cuando obedecen las instrucciones de un juez. Es inadmisible, pues, que un sindicato ampare esa conducta. Porque incitar a que los agentes desobedezcan a sus mandos es una auténtica subversión del Estado de Derecho. El SUP debería recapacitar y retirar esta absurda promesa". El 14 de diciembre, el colectivo de cerrajeros de Navarra anuncia que no colaborarán en ningún desahucio y anima al resto de sectores implicados en el proceso a que se animen a la iniciativa. 
el PSOE, aunque finalmente la negociación no fructifica y la leve reforma se aprueba mediante decreto ley.

Una vez que los desahucios se han convertido en issue, cada uno de los medios lo aborda de manera distinta, presentándolo bajo diferentes encuadres en función del alineamiento ideológico de cada uno de ellos. Para El País, "los desahucios son una de las consecuencias más graves de la crisis económica. El decreto aprobado por el Gobierno es un primer paso ante la demanda social y judicial que debe tener una continuación urgente y profunda". Para El Mundo, "los desahucios constituyen un grave problema social que requiere soluciones. El PSOE, que no actuó cuando estaba gobernando, dificulta ahora el pacto con el PP para aliviar este drama. La lucha más eficaz contra los desahucios es colocar al país en condiciones de crecer".

Además de estas diferencias en cuanto a atribución de responsabilidad y propuesta de solución que reflejan los respectivos encuadres, cada uno de los medios construye un discurso propio bajo el que subyacen sus posicionamientos ideológicos, que identificamos a partir de las siguientes categorías:

a) Ejes temáticos: Drama social y reivindicación de la política (acuerdo) para $E l$ País vs. irresponsabilidad ciudadana y crecimiento económico para El Mundo.

b) Iniciativa: Del Gobierno para El Mundo, en tanto que El País defiende que éste se limita a reaccionar a una demanda social.

c) Herramientas de solución: Acuerdo político (El País) vs. crecimiento económico (El Mundo, que confiaría al PP).

La manera en que los dos principales partidos políticos gestionan y ponen fin al problema de los desahucios en España sugiere que estamos ante una nueva escenificación de lo que Meyer (2002) denominó "pseudopolítica", esto es, una representación puramente autorreferencial en la que los actores políticos debaten sobre la capacidad y la iniciativa de cada cual para tomar decisiones y abordar un problema dado, sin que eso se traduzca en acciones políticas. En el caso que nos ocupa, encontramos que no sólo PP y PSOE son incapaces de alcanzar un acuerdo, sino que el decreto ley aprobado por el gobierno de Rajoy tampoco satisface las expectativas ciudadanas, en la medida en que sólo casos en condiciones muy extremas podrán acogerse a la prórroga establecida.

No en vano, el número de desahucios (y de suicidios por tal causa) continúa en los meses siguientes y el issue se reactiva cuando, el 5 de febrero $^{6}$, la portavoz de la Plataforma de Afectados por la Hipoteca acude al Congreso de los Diputados y expone un duro discurso contra bancos y partidos políticos, que tuvo una importante repercusión en medios y redes sociales ${ }^{7}$. Tres días después se produce un nuevo suicidio que incrementa la presencia del tema en los medios y las movilizaciones sociales para exigir al gobierno la aprobación de una Iniciativa Legislativa Popular

\footnotetext{
${ }^{6}$ El debate en torno a los desahucios continúa hasta que en abril de 2013, el Gobierno aprueba, sólo con los votos del PP, la Ley de Medidas para la Protección a los Deudores, Reestructuración de la Deuda y Alquiler Social. Sin embargo, la segunda fase del proceso queda fuera de nuestro estudio al exceder los márgenes temporales en los que este documento se trabajó públicamente, en el marco del Coloquio Anual de la AE-IC 2013.

7 https://www.youtube.com/watch?v=LtNBnc6khHg.
} 
para la dación en pago, presentada meses antes por la PAH. El 12 de febrero, la Plataforma de Afectados por la Hipoteca presentaba en el Parlamento la citada Iniciativa Legislativa Popular para admitir a debate parlamentario la dación en pago, que venía respaldada por más de 1,4 millones de firmas ciudadanas y todos los grupos parlamentarios excepto el PP, que había anunciado su rechazo. El mismo día se presentaba otra propuesta ciudadana para proclamar los toros Bien Cultural, avalada por algo más de medio millón de firmas y que contaría con el apoyo del PP. En este contexto, la presión ciudadana se incrementa los días previos y, el mismo día que se presenta la ILP se mantiene una protesta social ante el Congreso durante toda la jornada y la PAH -que en esos días se había convertido en la protagonista de las redes sociales y medios audiovisuales por el discurso de su portavoz en el Congreso de los Diputados- convoca a los medios para una escenificación gráfica del apoyo ciudadano a la ILP que el gobierno del PP iba a rechazar. Durante el transcurso del día se produce un nuevo suicidio ante un desalojo inminente, el de un matrimonio de jubilados. Todo ello, con su consiguiente repercusión en las redes sociales, lleva al Partido Popular a cambiar sobre la marcha ${ }^{8}$ su posición inicial y admitir a trámite la ILP sobre la dación en pago, algo que, en cualquier caso, no garantiza más que el debate parlamentario.

La prensa de referencia recoge la rectificación del Gobierno en sus páginas del día posterior (13/02/2013), sin modificar de manera significativa los encuadres que hasta entonces había mantenido. Para El País, "la presión popular obliga a Rajoy a debatir la iniciativa antidesahucios" (portada 13/II/2013); para El Mundo, "el hecho de que 1,4 millones de españoles la avalen obliga al Parlamento a estudiarla, aunque luego se rechace" (en el editorial "Hay que oír a la calle, pero sin pagar por ello", 13/II/2013). Con esta rectificación del Gobierno, que no supone en todo caso una modificación legislativa, el issue vuelve a desaparecer del foco mediático.

El análisis de este tema ilustra, asimismo, la interrelación que se establece entre los poderes social, político y mediático, aportando evidencia empírica a la tesis de que los poderes políticos muestran una mayor predisposición a tomar medidas cuando un tema es abordado por los medios de comunicación (Soroka, 2007: 190 s.), más aun cuando la materia en cuestión cuenta con una opinión pública homogénea y movilizada, como es en el caso de los desahucios.

\section{REFERENCIAS BIBLIOGRÁFICAS}

Bouza, Fermín y GonzÁLez, Juan Jesús (2009): Las razones del voto en la España democrática: 1977-2008. Madrid: Los Libros de la Catarata.

CAstromil, A. (2012): "Negativismo mediático y campaña electoral en las Elecciones Generales de 2008. Revista Española de Investigaciones Sociológicas, 139, pp. 163-174.

\footnotetext{
${ }^{8}$ Los diputados del PP habían advertido hasta esa misma mañana que no admitirían a debate la ILP relativa a los desahucios.
} 
Chavero, Palmira (2012): El papel de la agenda de los medios en el proceso de comunicación política. Un estudio de caso: la legislatura 2008-2011. Tesis doctoral. Madrid: Universidad Complutense de Madrid.

Entman, Robert (2004): Projections of power. Framing news, public opinion and US foreign policy, Chicago \& London: The University Chicago Press.

GonzÁlez, Juan Jesús y Chavero, Palmira (2012): "Política en portada: la construcción mediática de la crisis", en Colino, C. y Cotarelo, R. (eds.). España en crisis. Balance de la segunda legislatura de Rodríguez Zapatero. Valencia: Tirant Humanidades, pp. 289-310.

GONZÁLEZ, Juan Jesús y CHAVERO, Palmira (2013): "Variantes de negativismo informativo en un contexto de crisis global: la evaluación de los actores políticos y de su interrelación". Partidos, medios y electores en proceso de cambio. Las Elecciones Generales españolas de 2011. Valencia: Tirant lo Blanch, pp. 425-439.

Hallin, Daniel y Mancini, Paolo (2004): Comparing Media Systems. Three Models of Media and Politics, Cambridge: Cambridge University Press.

McCombs, Maxwell (2004): Estableciendo la agenda. Barcelona: Paidós Comunicación.

Meyer, Thomas (2002): Media Democracy. How the Media Colonize Politics. Cambridge: Polity Press.

Muñoz-Alonso, Alejandro y Juan Ignacio RosPIR. (1995): Comunicación política. Madrid: Editorial Universitas.

ReIG, Ramón. (1998): Medios de comunicación y poder en España. Barcelona: Paidós.

SOROKA, Stuart (2007): “Agenda-setting and Issue Definition”. En Orsini, M. y Smith, M. (eds.): Reconfiguring Policy Analysis: Contemporary Canadian Approaches, University of British Columbia Press.

VAN DiJK, Teun A. (2009): Discurso y poder, Barcelona: Editorial Gedisa.

- (1996): “Opiniones e ideologías en la prensa", Voces y culturas, pp. 9-50. 


\begin{abstract}
RESUMEN
Dadas las características del sistema mediático español, Hallin y Mancini (2004) lo sitúan en el denominado modelo de Pluralismo Polarizado. Uno de sus principales rasgos es la estrecha relación que se establece entre el sistema político y los medios de comunicación, que provoca que en ocasiones éstos primen intereses partidistas.

Para comprobar si los medios de comunicación se limitan a una función de mediadores o si, por el contrario, toman parte en el proceso político, en este trabajo analizamos el papel que ha desempeñado la prensa de referencia en uno de los temas del debate público actual: los desahucios. Para ello, analizamos El País y El Mundo desde el 1 de octubre hasta el 31 de diciembre de 2012, periodo en el que se activa el debate en la esfera pública y se realiza la primera modificación de la ley regulatoria. Esto nos permite analizar cómo se produce la aparición de un tema (desahucios) en la agenda mediática para desactivar la alarma social, la manera de abordarlos por parte de la prensa y su desaparición de la agenda mediática.
\end{abstract}

Palabras clave: polarización; agenda mediática; framing; noticiabilidad; desahucio.

\title{
Evictions in the Spanish press: different stories about public issues
}

\begin{abstract}
According to Hallin and Mancini (2004), Spain is part of a typology of media system called Polarized Pluralism. One of his main features is a close relation between media and politic system, which produces very political media.

In this paper we analyze how Spanish press has informed about one of the most important items in the public sphere nowadays: evictions. We study how El País and El Mundo have informed about it between $1^{\text {st }}$ October and $31^{\text {st }}$ December 2012, period in which the government modified the law about evictions. It allows us to know how an issue appears in media to silent the social protests, how journals inform about it and how the issue disappears from the public sphere.
\end{abstract}

Keywords: Polarization; media agenda; framing; issue; eviction. 